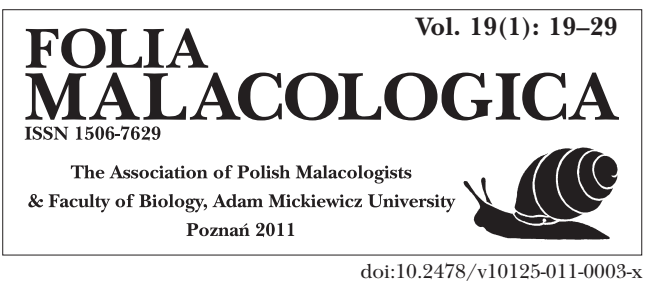

\title{
MOLLUSCS OF THE KRUTYNIA RIVER (MASURIAN LAKELAND)
}

\author{
BEATA JAKUBIK, KRZYSZTOF LEWANDOWSKI
}

\begin{abstract}
Institute of Biology, University of Natural Sciences and Humanities in Siedlce, B. Prusa 12, 08-110 Siedlce, Poland (e-mail: ekologia@ap.siedlce.pl)

ABSTRACT: Studies on molluscs of the Krutynia River (Masurian Lakeland) carried out in 2008-2009 (July) revealed the presence of 38 species (21 species of snails and 17 species of bivalves). The number of species per site ranged from 4 (middle section) to 27 (mouth section of the river). Snails Bithynia tentaculata, Viviparus contectus, Radix balthica and bivalves Sphaerium corneum, Dreissena polymorpha, Anodonta anatina, Unio pictorum and $U$. tumidus were found along the whole river course. The highest densities that locally reached 200 indiv. $/ \mathrm{m}^{2}$ were noted for Theodoxus fluviatilis and the largest biomass of over $2.5 \mathrm{~kg} / \mathrm{m}^{2}$ - for the unionid bivalves, mainly for Unio tumidus.
\end{abstract}

KEY WORDS: river, molluscs, long-term changes

\section{INTRODUCTION}

The Krutynia - one of the main rivers in the Masurian Lakeland - was the object of numerous physical, chemical and biological studies (e. g. HILLBRICHT-ILKOWSKA \& WIŚNIEWSKI 1996). Previous malacological studies on the river were done fifty to almost a hundred years ago (HILBERT 1913, BERGER 1960) or pertained to only small stretches of the river (LEWANDOWSKI 1996b, JAKUBIK \&

\section{MATERIAL AND METHODS}

The Krutynia is a medium-sized river, about 100 $\mathrm{km}$ long. It springs from Lake Warpuny, flows through 17 lakes and ends its course in Lake Bełdany (BAJKIEWICZ-GRABOWSKA 1996). Molluscs were sampled in 2008-2009 (in July) in 10 sites located in the river stretches between the lakes, from the springs to the mouth (Fig. 1). Diversity of lotic stretches of the Krutynia was considered when selecting the sampling sites. The sites, situated at a different distance from the lakes, differed in the width of the river, kind of substratum and plant cover (Table 1). Approximate cover of the river bottom by vegetation was estimated using the three-degree scale of BERNATOWICZ \& WOLNY (1974).
LEWANDOWSKI 2008), or else considered only a part of the species (BERGER 1962, PIECHOCKI 1989, LEWANDOWSKI 1996a). The aim of this study was to relate the qualitative and quantitative aspects of the malacofauna of the whole Krutynia River to the environmental variability, and to compare the present data with the earlier results.

In every site the bottom sediments were sampled to determine the content of total phosphorus with the molybdenum blue method and of total nitrogen with the indophenyl blue method (MARCZENKO 1979) after preliminary mineralisation according to the standard Kjeldahl procedure. Quantitative samples of molluscs were taken with a frame of an area of $0.25 \mathrm{~m}^{2}$ randomly placed on the bottom in four replicates in every sampling site. Biological material was removed from within the frame with a grab and washed on a benthic sieve of $1 \mathrm{~mm}$ mesh. Live molluscs and empty shells were collected. Apart from quantitative samples, qualitative samples were also collected in 2009 to estimate the microhabitat species richness. 


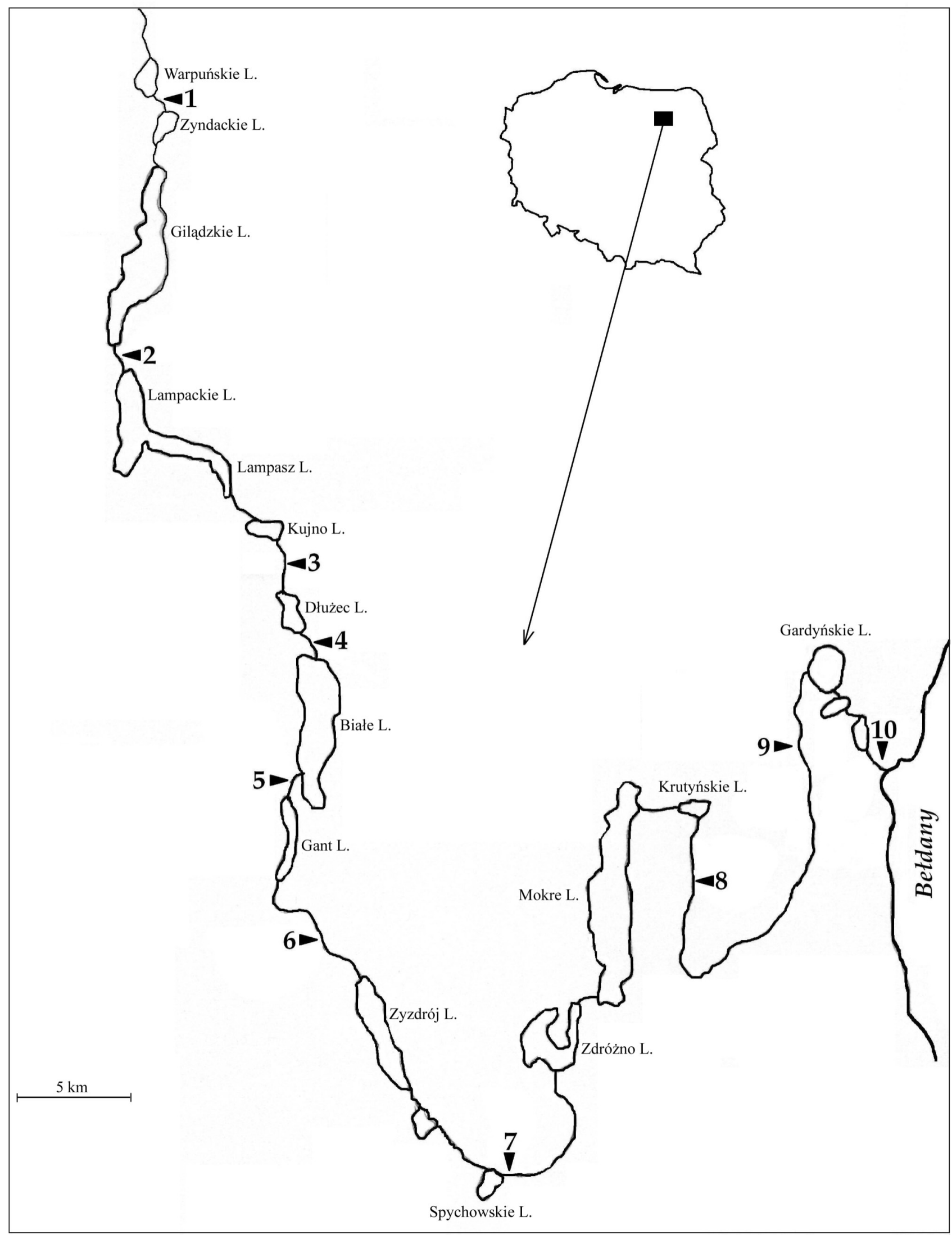

Fig. 1. Distribution of sampling sites (1 - Zyndaki, 2 - Sorkwity, 3 - Borowe, 4 - Dłużec, 5 - Gant, 6 - Machary, 7 - Spychowo, 8 - Wojnowo, 9 - Nowy Most, 10 - Iznota) 
Table 1. Characteristics of the study sites; plant coverage estimated according to the scale of BERNATOWICZ \& WOLNY (1974), where: 1 - very high density, 2 - medium density, 3 - low density

\begin{tabular}{|c|c|c|c|c|c|c|}
\hline No. & $\begin{array}{l}\text { River } \\
\text { width } \\
(\mathrm{m})\end{array}$ & $\begin{array}{l}\text { Maximum } \\
\text { depth } \\
\quad(\mathrm{m})\end{array}$ & $\begin{array}{l}\text { Distance } \\
\text { from the } \\
\text { river outlet } \\
\text { from a lake } \\
\quad(\mathrm{m})\end{array}$ & Type of bottom & $\begin{array}{l}\text { Approximate } \\
\text { plant cover } \\
{[\%]}\end{array}$ & Dominant plant species \\
\hline 1 & $2-3$ & 0.5 & 50 & sand, gravel, stones & $40[2]$ & $\begin{array}{l}\text { Nuphar lutea, Sparganium ramosum, } \\
\text { Potamogeton perfoliatus }\end{array}$ \\
\hline 2 & 8 & 0.5 & 100 & sand, gravel, stones & $20[2]$ & N. lutea, Acorus calamus, Phragmites australis \\
\hline 3 & $10-12$ & 0.5 & 500 & sand, gravel & $80[1]$ & N. lutea, S. ramosum \\
\hline 4 & $12-15$ & 0.5 & 100 & gravel, stones & $30[2]$ & Typha latifolia \\
\hline 5 & 12 & 1.0 & 200 & sand, stones & $30[2]$ & N. lutea, P. australis \\
\hline 6 & $12-15$ & 1.0 & 2000 & sand, gravel & $30[2]$ & N. lutea, P. australis \\
\hline 7 & 15 & 0.5 & 100 & gravel & no plants & - \\
\hline 8 & 20 & 1.0 & 2000 & sand, stones, mud & $30[2]$ & Carex sp., Utricularia vulgaris \\
\hline 9 & 15 & 1.0 & 25000 & gravel, stones, mud & $80[1]$ & $\begin{array}{l}\text { N. lutea, P. australis, A. calamus, Carex sp., } \\
\text { Sagittaria sagittifolia, Fontinalis antipyretica, } \\
\text { Ceratophyllum demersum }\end{array}$ \\
\hline 10 & $12-20$ & 1.0 & 200 & sand, mud & $20[2]$ & N. lutea, $P$. australis \\
\hline
\end{tabular}

Bivalves of the family Unionidae were determined to species level, their age was assessed and size was measured in the field, and then live bivalves were released. Other live molluscs were transported to the laboratory, identified and weighed as fast as possible. Most live molluscs were then released into the river. Only a small part of the material was preserved in $50 \%$ alcohol. Fresh biomass of unionid bivalves was calculated, considering species composition and size structure based on rich data from 1989 (LEWANDOWSKI 1996a).

The dominance index was calculated according to the formula: $\mathrm{D}=100 \mathrm{~S}_{\mathrm{a}} / \mathrm{S}$, where $\mathrm{S}_{\mathrm{a}}$ - sum of all individuals of a given species in all samples, $S$ - sum of individuals of all species in all samples. Frequency was calculated with the formula: $\mathrm{C}=100 \mathrm{n}_{\mathrm{a}} / \mathrm{N}$, where $\mathrm{n}_{\mathrm{a}}$ number of samples with species a, $\mathrm{N}-$ number of all samples. Similarity of species composition S (MARCZEWSKI \& STEINHAUS 1959) between particular sites was estimated with the equation: $\mathrm{S}=\mathrm{w} /(\mathrm{a}+\mathrm{b}-\mathrm{w})$, where a, b- number of species in sites A and B, respectively, $\mathrm{w}$ - number of species common to sites A and B. Species diversity was determined from the Shannon-Weaver index (MARGALEF 1958): $H^{\prime}=-\sum p_{i} l n p_{i}$, where $p_{i}$ - relative abundance of the $i^{\text {th }}$ species.

Normality of distribution was tested with the Shapiro-Wilk test (ŁOMNICKI 1995). To compare temporal changes in the concentration of nitrogen, phosphorus and organic matter, in the number of species, their density and biomass and the species diversity index we used non-parametric Wilcoxon's test (ŁOMNICKI 1995). Spearman linear correlation was used to test the relationship between the species diversity index and the number of species and their biomass. ANOVA was used to test the effect of the concentration of nitrogen, phosphorus and organic matter, of the bottom coverage by vegetation, type of the bottom and the distance from a lake on the number of species.

A total of 38 mollusc species were recorded (21 species of snails and 17 species of bivalves) (Tables 3 and 4). No significant differences were found in the number of species between the years (Wilcoxon test, $\mathrm{N}=10, \mathrm{Z}=0.49, \mathrm{p}=0.624)$. Bithynia tentaculata, Viviparus contectus, Radix balthica, Sphaerium corneum, Dreissena polymorpha, Anodonta anatina, Unio pictorum and $U$. tumidus were noted along the whole river length. Common species, found only in some river sections, were: Theodoxus fluviatilis (not found in the middle trogen (Wilcoxon test, $\mathrm{N}=40, \mathrm{Z}=0.36, \mathrm{p}=0.722$ ) or organic matter (Wilcoxon test, $\mathrm{N}=20, \mathrm{Z}=0.84, \mathrm{p}=0.401$ ). 
Table 2. Chemical characteristics of bottom sediments in the Krutynia in 2008-2009

\begin{tabular}{ccccccc}
\hline \multirow{2}{*}{ Sites } & \multicolumn{2}{c}{$\mathrm{N}(\mathrm{mg} / \mathrm{g}$ dry wt. } & \multicolumn{2}{c}{$\mathrm{P}(\mathrm{mg} / \mathrm{g}$ dry wt. } & \multicolumn{2}{c}{ Organic matter (\% dry wt.) } \\
\cline { 2 - 6 } & 2008 & 2009 & 2008 & 2009 & 2008 & 2009 \\
\hline 1 & $0.34-0.66$ & $0.47-0.65$ & $0.22-0.30$ & $0.25-0.31$ & $1.11-1.16$ & $1.25-1.49$ \\
2 & $0.21-1.02$ & $0.27-0.59$ & $0.44-0.55$ & $0.33-0.46$ & $1.01-1.43$ & $1.21-1.50$ \\
3 & $0.18-0.59$ & $0.28-0.40$ & $0.27-0.45$ & $0.20-0.26$ & $0.91-1.02$ & $1.07-1.15$ \\
4 & $0.31-0.53$ & $0.52-0.78$ & $0.39-0.52$ & $0.30-0.34$ & $1.54-1.74$ & $1,47-2.21$ \\
5 & $0.17-0.66$ & $0.24-0.26$ & $0.30-0.57$ & $0.21-0.27$ & $0.60-1.50$ & $0.67-0.83$ \\
6 & $0.23-0.45$ & $0.22-0.24$ & $0.25-0.33$ & $0.23-0.27$ & $1.01-1.07$ & $0.57-0.66$ \\
7 & $0.10-0.23$ & $0.23-0.42$ & $0.32-0.42$ & $0.33-0.44$ & $0.59-0.67$ & $1.12-1.40$ \\
8 & $0.39-1.15$ & $0.30-0.35$ & $0.32-0.38$ & $0.20-0.25$ & $1.63-2.97$ & $0.87-1.03$ \\
9 & $0.53-1.19$ & $0.75-0.92$ & $0.44-0.49$ & $0.35-0.59$ & $3.10-7.00$ & $22.2-29.7$ \\
10 & $0.12-0.39$ & $0.40-0.43$ & $0.28-0.38$ & $0.35-0.47$ & $0.60-0.68$ & $0.29-1.07$ \\
\hline
\end{tabular}

section) and Potamopyrgus antipodarum (present in the upper and middle sections). The least frequent were Viviparus viviparus, Stagnicola palustris, S. corvus, Planorbis planorbis, P. carinatus, Acroloxus lacustris, Pseudanodonta complanata, Sphaerium rivicola, Pisidium crassum and Musculium lacustre. Only empty shells of Gyraulus albus were found. Dominant species were: $T$. fluviatilis (c. 26\%), B. tentaculata (c. 20\%) and $U$.

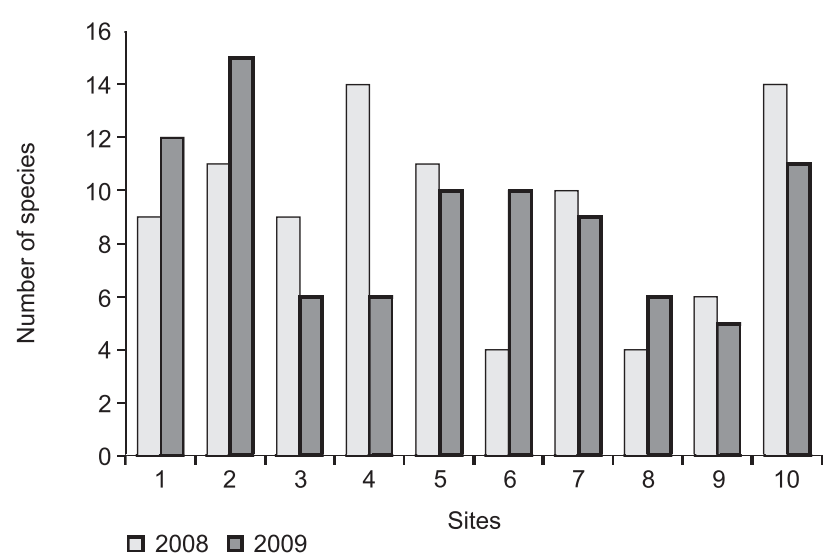

Fig. 2. Number of mollusc species in particular sites of the Krutynia

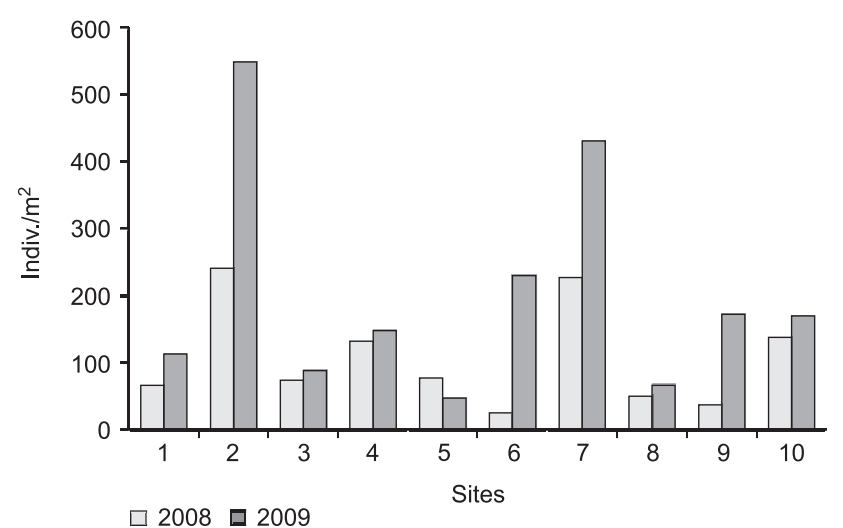

Fig. 3. Density (indiv. $/ \mathrm{m}^{2}$ ) of molluscs in particular sites of the Krutynia tumidus (c. 13\%) (Table 3). The number of species in individual sites ranged from 4 to 15 (Fig. 2).

Theodoxus fluviatilis reached the highest densities, locally exceeding $200 \mathrm{indiv}$. $/ \mathrm{m}^{2}$. From among bivalves Dreissena polymorpha formed populations of the density of over 40 indiv. $/ \mathrm{m}^{2}$, like Sphaerium corneum. Relatively high densities were observed for unionid bivalves. Unio tumidus reached the density of several dozen indiv. $/ \mathrm{m}^{2}$ (maximum 65 indiv. $/ \mathrm{m}^{2}$ ) in the middle and lower sections of the river. The presence of Anodonta cygnea in both lower and upper parts of the river, at a density of 12 indiv. $/ \mathrm{m}^{2}$, is noteworthy. Total mollusc densities in particular sites ranged from 25 indiv. $/ \mathrm{m}^{2}$ (site 6 in 2008) to ca. 550 indiv. $/ \mathrm{m}^{2}$ (site 2 in 2009) (Fig. 3). Biomass varied from c. 13 to over $2,500 \mathrm{~g} / \mathrm{m}^{2}$ (Fig. 4). In the latter case, the large biomass was associated with unionid bivalves which comprised over $99 \%$ of the total biomass. Adult individuals of a length between 6 and $7 \mathrm{~cm}$ dominated in this group of bivalves, and the age of the oldest individuals was estimated at 7-9 years. No significant differences were found in density (Wilcoxon test, $\mathrm{N}=10, \mathrm{Z}=2.39$, $\mathrm{p}=0.017$ ) and biomass (Wilcoxon test, $\mathrm{N}=10, \mathrm{Z}=0.05$, $\mathrm{p}=0.959$ ) between the two study years.

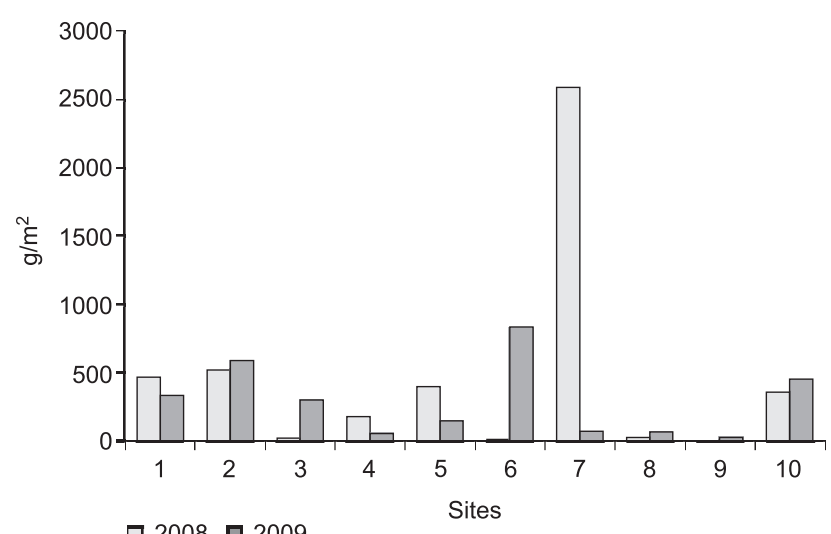

Fig. 4. Biomass $\left(\mathrm{g} / \mathrm{m}^{2}\right)$ of molluscs in particular sites of the Krutynia 
Table 3. Molluscs recorded from the Krutynia in 2008-2009; 0 - empty shells

\begin{tabular}{|c|c|c|c|c|c|c|c|}
\hline \multirow[b]{2}{*}{ No. } & \multirow[b]{2}{*}{ Species } & \multicolumn{3}{|c|}{2008} & \multicolumn{3}{|c|}{2009} \\
\hline & & $\mathrm{N}$ & $\begin{array}{c}\text { Frequency } \\
(\%)\end{array}$ & $\begin{array}{c}\text { Dominance } \\
(\%)\end{array}$ & $\mathrm{N}$ & $\begin{array}{c}\text { Frequency } \\
(\%)\end{array}$ & $\begin{array}{c}\text { Dominance } \\
(\%)\end{array}$ \\
\hline \multicolumn{8}{|c|}{ Gastropoda } \\
\hline 1 & Theodoxus fluviatilis (Linnaeus, 1758) & 278 & 50 & 25.8 & 462 & 50 & 26.3 \\
\hline 2 & Viviparus viviparus (Linnaeus, 1758) & - & - & - & 6 & 10 & 0.3 \\
\hline 3 & Viviparus contectus (Millet, 1813) & 44 & 60 & 4.1 & 36 & 40 & 2.1 \\
\hline 4 & Valvata piscinalis (O. F. Müller, 1774) & 3 & 20 & 0.3 & 38 & 50 & 2.2 \\
\hline 5 & Potamopyrgus antipodarum (J. E. Gray, 1843) & 83 & 50 & 7.7 & 278 & 60 & 15.8 \\
\hline 6 & Bithynia tentaculata (Linnaeus, 1758) & 217 & 90 & 20.1 & 300 & 80 & 17.1 \\
\hline 7 & Galba truncatula (O. F. Müller, 1774) & - & - & - & 2 & 10 & 0.1 \\
\hline 8 & Stagnicola palustris (O. F. Müller, 1774) & 1 & 10 & 0.1 & - & - & - \\
\hline 9 & Stagnicola corvus (Gmelin, 1791) & - & - & - & 8 & 10 & 0.5 \\
\hline 10 & Radix auricularia (Linnaeus, 1758) & 11 & 30 & 1.0 & 14 & 20 & 0.8 \\
\hline 11 & Radix balthica (Linnaeus, 1758) & 17 & 60 & 1.5 & 12 & 30 & 0.7 \\
\hline 12 & Lymnaea stagnalis (Linnaeus, 1758) & 6 & 40 & 0.5 & 18 & 30 & 1.0 \\
\hline 13 & Planorbis planorbis (Linnaeus, 1758) & 1 & 10 & 0.1 & - & - & - \\
\hline 14 & Planorbis carinatus O. F. Müller, 1774 & 2 & 10 & 0.2 & - & - & - \\
\hline 15 & Anisus leucostomus (Millet, 1813) & 3 & 30 & 0.3 & - & - & - \\
\hline 16 & Anisus vortex (Linnaeus, 1758) & - & - & - & 12 & 20 & 0,7 \\
\hline 17 & Bathyomphalus contortus (Linnaeus, 1758) & 2 & 20 & 0.2 & - & - & - \\
\hline 18 & Gyraulus albus (O. F. Müller, 1774) & 0 & - & - & 0 & - & - \\
\hline 19 & Planorbarius corneus (Linnaeus, 1758) & 20 & 40 & 1.8 & 6 & 20 & 0.3 \\
\hline 20 & Ancylus fluviatilis O. F. Müller, 1774 & 13 & 20 & 1.2 & - & - & - \\
\hline 21 & Acroloxus lacustris (Linnaeus, 1758) & - & - & - & 4 & 10 & 0.2 \\
\hline \multicolumn{8}{|c|}{ Bivalvia } \\
\hline 22 & Unio pictorum (Linnaeus, 1758) & 26 & 40 & 2.4 & 27 & 60 & 1.5 \\
\hline 23 & Unio tumidus Philipsson, 1788 & 138 & 40 & 12.8 & 223 & 60 & 12.7 \\
\hline 24 & Unio crassus Philipsson, 1788 & - & - & - & 6 & 30 & 0.3 \\
\hline 25 & Anodonta cygnea (Linnaeus, 1758) & 8 & 20 & 0.7 & 14 & 20 & 0.8 \\
\hline 26 & Anodonta anatina (Linnaeus, 1758) & 62 & 60 & 5.8 & 42 & 50 & 2.4 \\
\hline 27 & Pseudanodonta complanata (Rossmässler, 1835) & 4 & 10 & 0.4 & - & - & - \\
\hline 28 & Dreissena polymorpha (Pallas, 1771) & 71 & 50 & 6.6 & 54 & 40 & 3.1 \\
\hline 29 & Sphaerium corneum (Linnaeus, 1758) & 44 & 50 & 4.1 & 158 & 80 & 9.0 \\
\hline 30 & Sphaerium rivicola (Lamarck, 1818) & 1 & 10 & 0.1 & - & - & - \\
\hline 31 & Musculium lacustre (O. F. Müller, 1774) & - & - & - & 2 & 10 & 0.1 \\
\hline 32 & Pisidium amnicum (O. F. Müller, 1774) & 3 & 30 & 0.3 & 10 & 10 & 0.6 \\
\hline 33 & Pisidium henslowanum (Sheppard, 1823) & 4 & 20 & 0.4 & 6 & 30 & 0.3 \\
\hline 34 & Pisidium supinum A. Schmidt, 1851 & 2 & 10 & 0.2 & 2 & 10 & 0.1 \\
\hline 35 & Pisidium subtruncatum Malm, 1855 & 1 & 10 & 0.1 & 6 & 30 & 0.3 \\
\hline 36 & Pisidium nitidum Jenyns, 1832 & 4 & 10 & 0.4 & 4 & 10 & 0.2 \\
\hline 37 & Pisidium crassum Stelfox, 1918 & 4 & 10 & 0.4 & - & - & - \\
\hline 38 & Pisidium casertanum (Poli, 1791) & 4 & 10 & 0.4 & 8 & 20 & 0.5 \\
\hline
\end{tabular}


Table 4. Molluscs recorded from particular sites in the Krutynia in 2008-2009; $\times$ - live molluscs, o - empty shells

\begin{tabular}{|c|c|c|c|c|c|c|c|c|c|c|c|c|}
\hline \multirow{2}{*}{ No. } & \multirow{2}{*}{ Species } & \multirow{2}{*}{ Year } & \multicolumn{10}{|c|}{ Sites } \\
\hline & & & 1 & 2 & 3 & 4 & 5 & 6 & 7 & 8 & 9 & 10 \\
\hline \multicolumn{13}{|c|}{ Gastropoda } \\
\hline \multirow[t]{2}{*}{1} & Theodoxus fluviatilis & 2008 & & $x$ & & & & & $x$ & $\times$ & $x$ & $x$ \\
\hline & & 2009 & & $x$ & & & & & $\times$ & $x$ & $x$ & $x$ \\
\hline \multirow[t]{2}{*}{2} & Viviparus viviparus & 2008 & & & & & & & o & & & o \\
\hline & & 2009 & & & & & & & & & & $x$ \\
\hline \multirow[t]{2}{*}{3} & $V$. contectus & 2008 & & $x$ & $x$ & $x$ & $x$ & & o & $x$ & o & $x$ \\
\hline & & 2009 & & $x$ & $x$ & & & $x$ & o & $\times$ & & \\
\hline \multirow[t]{2}{*}{4} & Valvata piscinalis & 2008 & & & $x$ & & $x$ & & & & & \\
\hline & & 2009 & $x$ & $x$ & $x$ & $x$ & $x$ & & & & & o \\
\hline \multirow[t]{2}{*}{5} & Potamopyrgus antipodarum & 2008 & $x$ & $x$ & & $\times$ & $x$ & & $x$ & & & \\
\hline & & 2009 & $x$ & $x$ & & $x$ & $x$ & $x$ & $x$ & & & \\
\hline \multirow[t]{2}{*}{6} & Bithynia tentaculata & 2008 & $x$ & $x$ & $x$ & $\times$ & $\times$ & $\times$ & & $\times$ & $\times$ & $\times$ \\
\hline & & 2009 & $x$ & $x$ & $x$ & $x$ & $x$ & $x$ & & o & $x$ & $x$ \\
\hline \multirow[t]{2}{*}{7} & Galba truncatula & 2008 & & & & & & & & & & \\
\hline & & 2009 & & $x$ & & & & & & & & \\
\hline \multirow[t]{2}{*}{8} & Stagnicola palustris & 2008 & & & & & & & & & $x$ & \\
\hline & & 2009 & & & & & & & & & & \\
\hline \multirow[t]{2}{*}{9} & S. corvus & 2008 & & & & & & & & & & \\
\hline & & 2009 & $x$ & & & $\times$ & $\times$ & & & o & & \\
\hline \multirow[t]{2}{*}{10} & Radix auricularia & 2008 & & & & $\times$ & $\times$ & & $\times$ & & & \\
\hline & & 2009 & & & o & $x$ & o & $x$ & & & & o \\
\hline \multirow[t]{2}{*}{11} & R. balthica & 2008 & $x$ & & & $x$ & & $x$ & $x$ & & $x$ & $x$ \\
\hline & & 2009 & $\times$ & & & & & & & $x$ & & $x$ \\
\hline \multirow[t]{2}{*}{12} & Lymnaea stagnalis & 2008 & $\times$ & $x$ & & $x$ & & & & & & $x$ \\
\hline & & 2009 & $x$ & & & & & $x$ & & $\times$ & & o \\
\hline \multirow[t]{2}{*}{13} & Planorbis planorbis & 2008 & & & & $\times$ & & & & & & o \\
\hline & & 2009 & & & & & & & & & & \\
\hline \multirow[t]{2}{*}{14} & P. carinatus & 2008 & & & & & & & & & & $x$ \\
\hline & & 2009 & o & o & & o & o & & & & & \\
\hline \multirow[t]{2}{*}{15} & Anisus leucostomus & 2008 & & & $x$ & & & $x$ & & & $x$ & o \\
\hline & & 2009 & & & & & & & & & & \\
\hline 16 & A. vortex & 2008 & & & & & & & & & & \\
\hline & & 2009 & & & & o & & & & $\times$ & $x$ & o \\
\hline 17 & Bathyomphalus contortus & 2008 & & & $x$ & $\times$ & & & & & & \\
\hline & & 2009 & o & o & & & o & & & & & \\
\hline 18 & Gyraulus albus & 2008 & & $\mathrm{o}$ & & & & & & & & \\
\hline & & 2009 & o & o & & & & & & & & \\
\hline 19 & Planorbarius corneus & 2008 & $\times$ & & & $x$ & & $x$ & & o & & $x$ \\
\hline & & 2009 & $x$ & & & & $x$ & & o & o & & \\
\hline 20 & Ancylus fluviatilis & 2008 & $\times$ & & & & $x$ & & & & & \\
\hline & & 2009 & & & & & & & & & & \\
\hline 21 & Acroloxus lacustris & 2008 & & & & & & & & & & \\
\hline & & 2009 & & & & & & & & & & $x$ \\
\hline
\end{tabular}




\begin{tabular}{|c|c|c|c|c|c|c|c|c|c|c|c|c|}
\hline \multirow{2}{*}{ No. } & \multirow{2}{*}{ Species } & \multirow{2}{*}{ Year } & \multicolumn{10}{|c|}{ Sites } \\
\hline & & & 1 & 2 & 3 & 4 & 5 & 6 & 7 & 8 & 9 & 10 \\
\hline \multicolumn{13}{|c|}{ Bivalvia } \\
\hline \multirow[t]{2}{*}{22} & Unio pictorum & 2008 & o & $\times$ & & $x$ & & & $x$ & & & $x$ \\
\hline & & 2009 & o & $x$ & & $\times$ & $x$ & $\times$ & $\times$ & & & $x$ \\
\hline \multirow[t]{2}{*}{23} & U. tumidus & 2008 & & & & $x$ & $x$ & & $\times$ & o & & $x$ \\
\hline & & 2009 & $x$ & $x$ & o & & $x$ & $x$ & $x$ & & & $x$ \\
\hline \multirow[t]{2}{*}{24} & $U$. crassus & 2008 & & & & & & & & & & \\
\hline & & 2009 & & $x$ & & & $x$ & & $x$ & & & \\
\hline \multirow[t]{2}{*}{25} & Anodonta cygnea & 2008 & $x$ & $x$ & & & & & & o & & \\
\hline & & 2009 & $x$ & & $x$ & & & & o & & & \\
\hline \multirow[t]{2}{*}{26} & A. anatina & 2008 & $x$ & $x$ & o & $x$ & $\times$ & & $x$ & & & $x$ \\
\hline & & 2009 & $\times$ & $x$ & o & o & $x$ & $x$ & & & & $x$ \\
\hline \multirow[t]{2}{*}{27} & Pseudanodonta complanata & 2008 & & & & & & & & & & $x$ \\
\hline & & 2009 & & & & & & & o & & & \\
\hline \multirow[t]{2}{*}{28} & Dreissena polymorpha & 2008 & & $x$ & $x$ & $x$ & $x$ & & $x$ & & & \\
\hline & & 2009 & $x$ & $x$ & & & & o & $x$ & & & $x$ \\
\hline \multirow[t]{2}{*}{29} & Sphaerium corneum & 2008 & $x$ & & $x$ & & & & $x$ & $x$ & & $\times$ \\
\hline & & 2009 & $\times$ & $x$ & $x$ & $x$ & $x$ & & $x$ & & $x$ & $x$ \\
\hline \multirow[t]{2}{*}{30} & S. rivicola & 2008 & & & & & & & & & $x$ & \\
\hline & & 2009 & & & & & & & & & & \\
\hline \multirow[t]{2}{*}{31} & Musculium lacustre & 2008 & & & & & & & & & & \\
\hline & & 2009 & $x$ & & & & & & & & & \\
\hline \multirow[t]{2}{*}{32} & Pisidium amnicum & 2008 & & $x$ & $x$ & & $x$ & & & & & \\
\hline & & 2009 & & & & & & $\times$ & & & & $\mathrm{o}$ \\
\hline \multirow[t]{2}{*}{33} & P. henslowanum & 2008 & & & & & $x$ & & & & & $x$ \\
\hline & & 2009 & & $\times$ & & & $x$ & & & & & $\times$ \\
\hline \multirow[t]{2}{*}{34} & P. supinum & 2008 & & & & & & & $x$ & & & \\
\hline & & 2009 & & & & & & & $x$ & & & \\
\hline \multirow[t]{2}{*}{35} & P. subtruncatum & 2008 & & & & & & & & & & $x$ \\
\hline & & 2009 & & $x$ & & & & $x$ & $x$ & & & \\
\hline \multirow[t]{2}{*}{36} & P. nitidum & 2008 & & & $x$ & & & & & & & \\
\hline & & 2009 & & & $x$ & & & & & & & \\
\hline \multirow[t]{2}{*}{37} & P. crassum & 2008 & & & & $x$ & & & & & & \\
\hline & & 2009 & & & & & & & & & & \\
\hline \multirow[t]{2}{*}{38} & P. casertanum & 2008 & & $\times$ & & & & & & & & \\
\hline & & 2009 & & $\times$ & & & & & & & $x$ & \\
\hline
\end{tabular}

Species diversity was the greatest in site 2 in 2008 $\left(H^{\prime}=3.77\right)$ and in site 7 in $2009\left(H^{\prime}=4.68\right)$. The lowest Shannon-Weaver indices were noted in both years in site $5\left(H^{\prime}=1.19\right.$ and 1.74$)$. No statistically significant differences were found in the species diversity between the two study years $(\mathrm{N}=10, \mathrm{Z}=1.68$, $\mathrm{p}=0.093)$. There was no correlation between the species diversity index and the number of species (2008: $\left.\mathrm{r}_{\mathrm{s}}=0.38, \mathrm{p}=0.278 ; 2009: \mathrm{r}_{\mathrm{s}}=0.31, \mathrm{p}=0.387\right)$ and their biomass (2008: $r_{s}=0.46, p=0.174 ; 2009: r_{s}=-0.16$, $\mathrm{p}=0.651$ ).
Similarity indices calculated for 2008 showed the greatest similarity of species composition between sites 4 and $7(\mathrm{~S}=0.50)$ and the smallest between sites 2 and 6 and between 5 and $6(\mathrm{~S}=0.07)$ (Table 5). In 2009 the greatest similarity was found between sites 2 and $7(\mathrm{~S}=0.50)$ and no similarity - between sites 4 and 8 and 5 and 8 (Table 6 ).

Concentrations of nitrogen, phosphorus and organic matter in the bottom sediments did not affect the number of species in particular sites (ANOVA; $\left.\mathrm{F}_{3.16}=0.44, \mathrm{p}=0.727\right)$. The number of species did not 
Table 5. Similarity between sites in 2008

\begin{tabular}{|c|c|c|c|c|c|c|c|c|c|c|}
\hline & 1 & 2 & 3 & 4 & 5 & 6 & 7 & 8 & 9 & 10 \\
\hline 1 & $\mathrm{X}$ & 0.33 & 0.12 & 0.35 & 0.25 & 0.30 & 0.27 & 0.18 & 0.15 & 0.35 \\
\hline 2 & 0.33 & $\mathrm{X}$ & 0.33 & 0.47 & 0.37 & 0.07 & 0.31 & 0.25 & 0.13 & 0.31 \\
\hline 3 & 0.12 & 0.33 & $\mathrm{X}$ & 0.15 & 0.33 & 0.18 & 0.12 & 0.30 & 0.15 & 0.15 \\
\hline 4 & 0.35 & 0.47 & 0.15 & $\mathrm{X}$ & 0.39 & 0.20 & 0.50 & 0.12 & 0.11 & 0.33 \\
\hline 5 & 0.25 & 0.37 & 0.33 & 0.39 & $\mathrm{X}$ & 0.07 & 0.23 & 0.15 & 0.06 & 0.25 \\
\hline 6 & 0.30 & 0.07 & 0.18 & 0.20 & 0.07 & $\mathrm{X}$ & 0.08 & 0.14 & 0.43 & 0.20 \\
\hline 7 & 0.27 & 0.31 & 0.12 & 0.50 & 0.23 & 0.08 & $\mathrm{X}$ & 0.17 & 0.14 & 0.33 \\
\hline 8 & 0.18 & 0.25 & 0.30 & 0.12 & 0.15 & 0.14 & 0.17 & $\mathrm{X}$ & 0.25 & 0.28 \\
\hline 9 & 0.15 & 0.13 & 0.15 & 0.11 & 0.06 & 0.43 & 0.14 & 0.25 & $\mathrm{X}$ & 0.18 \\
\hline 10 & 0.35 & 0.31 & 0.15 & 0.33 & 0.25 & 0.20 & 0.33 & 0.28 & 0.18 & $\mathrm{X}$ \\
\hline
\end{tabular}

$<0.20 \quad 0.20-0.40>0.40$

Table 6. Similarity between sites in 2009

\begin{tabular}{|c|c|c|c|c|c|c|c|c|c|c|}
\hline & 1 & 2 & 3 & 4 & 5 & 6 & 7 & 8 & 9 & 10 \\
\hline 1 & $\mathrm{X}$ & 0.35 & 0.28 & 0.28 & 0.47 & 0.29 & 0.23 & 0.12 & 0.13 & 0.35 \\
\hline 2 & 0.35 & $\mathrm{X}$ & 0.23 & 0.31 & 0.35 & 0.39 & 0.50 & 0.10 & 0.25 & 0.44 \\
\hline 3 & 0.28 & 0.23 & $\mathrm{X}$ & 0.33 & 0.23 & 0.14 & 0.07 & 0.09 & 0.22 & 0.13 \\
\hline 4 & 0.28 & 0.31 & 0.33 & $\mathrm{X}$ & 0.45 & 0.33 & 0.25 & 0 & 0.22 & 0.21 \\
\hline 5 & 0.47 & 0.35 & 0.23 & 0.45 & $\mathrm{X}$ & 0.33 & 0.36 & 0 & 0.15 & 0.31 \\
\hline 6 & 0.29 & 0.39 & 0.14 & 0.33 & 0.33 & $\mathrm{X}$ & 0.27 & 0.17 & 0.07 & 0.17 \\
\hline 7 & 0.23 & 0.50 & 0.07 & 0.25 & 0.36 & 0.27 & $\mathrm{X}$ & 0.07 & 0.17 & 0.33 \\
\hline 8 & 0.12 & 0.10 & 0.09 & 0 & 0 & 0.17 & 0.07 & $\mathrm{X}$ & 0.22 & 0.13 \\
\hline 9 & 0.13 & 0.25 & 0.22 & 0.22 & 0.15 & 0.07 & 0.17 & 0.22 & $\mathrm{X}$ & 0.23 \\
\hline 10 & 0.35 & 0.44 & 0.13 & 0.21 & 0.31 & 0.17 & 0.33 & 0.13 & 0.23 & $\mathrm{X}$ \\
\hline
\end{tabular}

$<0.20 \quad 0.20-0.40>0.40$

depend on the degree of bottom coverage by vegetation (ANOVA; $\mathrm{F}_{3.9}=1.27, \mathrm{p}=0.423$ ) despite the fact that two extreme situations were represented (bottom almost wholly plant-covered as opposed to vegetation-free substratum), or on the type of substratum
(ANOVA; $\mathrm{F}_{2.9}=0.36, \mathrm{p}=0.723$ ). The only factor significantly affecting the number of species was the distance of the sampling site from a lake (ANOVA; $\left.\mathrm{F}_{1.9}=10.89, \mathrm{p}=0.046\right)$.

\section{DISCUSSION}

The species richness (38) and species composition of the malacofauna in the Krutynia recorded in 2008-2009 did not differ much from the earlier data. Fifty years previously, BERGER $(1960,1962)$ found five species more, i. e. 24 species of snails and 19 species of bivalves. A similar number of species (44) was reported for the beginning of the 20th century by HILBERT (1913). In his list HiLBERT (1913) included as separate species what is now regarded as forms or synonyms: Paludina diluvianiformis (a form of Viviparus contectus),

Valvata antiqua (a form of Valvata piscinalis), Limnaea ovata (or L. peregra - Radix balthica), Anodonta piscinalis (a synonym of $A$. anatina also recorded by that author), Sphärium mamillanum (a form of Sphaerium corneum). The actual number of species recorded by that author was thus only 39 (Table 7 ).

As many as 23 species were recorded in all three compared study periods, and another 11 found in 2008-2009 were also recorded in one of the earlier papers. It can be thus assumed that 33 mollusc species 
Table 7. Species composition of molluscs in the Krutynia according to various sources

\begin{tabular}{|c|c|c|c|c|}
\hline No. & Species & Hilbert 1913 & Berger 1960, 1962 & Own study \\
\hline \multicolumn{5}{|c|}{ Gastropoda } \\
\hline 1 & Theodoxus fluviatilis (Linnaeus, 1758) & $\times$ & $\times$ & $\times$ \\
\hline 2 & Viviparus viviparus (Linnaeus, 1758) & $x$ & $x$ & $x$ \\
\hline 3 & Viviparus contectus (Millet, 1813) & $x$ & $\times$ & $\times$ \\
\hline 4 & Valvata piscinalis (O. F. Müller, 1774) & $\times$ & $\times$ & $\times$ \\
\hline 5 & Valvata cristata O. F. Müller, 1774 & $\times$ & $x$ & \\
\hline 6 & Valvata macrostoma Mörch, 1864 & $\times$ & & \\
\hline 7 & Potamopyrgus antipodarum (J. E. Gray, 1843) & & & $\times$ \\
\hline 8 & Marstoniopsis scholtzi (A. Schmidt, 1856) & $\times$ & $\times$ & \\
\hline 9 & Bithynia tentaculata (Linnaeus, 1758) & $x$ & $\times$ & $\times$ \\
\hline 10 & Bithynia leachii (Sheppard, 1823) & $\times$ & $\times$ & \\
\hline 11 & Physa fontinalis (Linnaeus, 1758) & $x$ & $x$ & \\
\hline 12 & Galba truncatula (O. F. Müller, 1774) & $x$ & & $x$ \\
\hline 13 & Stagnicola palustris (O. F. Müller, 1774) & $x$ & & $\times$ \\
\hline 14 & Stagnicola corvus (Gmelin, 1791) & & & $\times$ \\
\hline 15 & Radix auricularia (Linnaeus, 1758) & $\times$ & $\times$ & $\times$ \\
\hline 16 & Radix balthica (Linnaeus, 1758) & $\times$ & $\times$ & $\times$ \\
\hline 17 & Radix ampla (W. Hartmann, 1821) & $x$ & & \\
\hline 18 & Myxas glutinosa (O. F. Müller, 1774) & & $x$ & \\
\hline 19 & Lymnaea stagnalis (Linnaeus, 1758) & $x$ & $\times$ & $\times$ \\
\hline 20 & Planorbis planorbis (Linnaeus, 1758) & $x$ & $x$ & $x$ \\
\hline 21 & Planorbis carinatus O. F. Müller, 1774 & $x$ & $x$ & $x$ \\
\hline 22 & Anisus leucostomus (Millet, 1813) & $x$ & & $\times$ \\
\hline 23 & Anisus vortex (Linnaeus, 1758) & $\times$ & $\times$ & $x$ \\
\hline 24 & Anisus vorticulus (Troschel, 1834) & & $\times$ & \\
\hline 25 & Anisus spirorbis (Linnaeus, 1758) & $\times$ & & \\
\hline 26 & Anisus septemgyratus (Rossmässler, 1835) & $\times$ & & \\
\hline 27 & Bathyomphalus contortus (Linnaeus, 1758) & $\times$ & & $\times$ \\
\hline 28 & Segmentina nitida (O. F. Müller, 1774) & $\times$ & & \\
\hline 29 & Gyraulus albus (O. F. Müller, 1774) & & $\times$ & $\times$ \\
\hline 30 & Gyraulus laevis (Alder, 1838) & & $x$ & \\
\hline 31 & Gyraulus crista (Linnaeus, 1758) & $x$ & $x$ & \\
\hline 32 & Hippeutis complanatus (Linnaeus, 1758) & $\times$ & $\times$ & \\
\hline 33 & Planorbarius corneus (Linnaeus, 1758) & $\times$ & $\times$ & $\times$ \\
\hline 34 & Ancylus fluviatilis O. F. Müller, 1774 & $\times$ & $\times$ & $x$ \\
\hline 35 & Acroloxus lacustris (Linnaeus, 1758) & & $\times$ & $\times$ \\
\hline \multicolumn{5}{|c|}{ Bivalvia } \\
\hline 36 & Unio pictorum (Linnaeus, 1758) & $\times$ & $\times$ & $\times$ \\
\hline 37 & Unio tumidus Philipsson, 1788 & $x$ & $\times$ & $\times$ \\
\hline 38 & Unio crassus Philipsson, 1788 & $\times$ & $\times$ & $x$ \\
\hline 39 & Anodonta cygnea (Linnaeus, 1758) & $x$ & $\times$ & $\times$ \\
\hline 40 & Anodonta anatina (Linnaeus, 1758) & $x$ & $\times$ & $\times$ \\
\hline 41 & Pseudanodonta complanata (Rossmässler, 1835) & $\times$ & $\times$ & $\times$ \\
\hline 42 & Dreissena polymorpha (Pallas, 1771) & $x$ & $\times$ & $x$ \\
\hline
\end{tabular}




\begin{tabular}{|c|c|c|c|c|}
\hline No. & Species & Hilbert 1913 & Berger 1960, 1962 & Own study \\
\hline 43 & Sphaerium corneum (Linnaeus, 1758) & $\times$ & $\times$ & $\times$ \\
\hline 44 & Sphaerium rivicola (Lamarck, 1818) & & & $\times$ \\
\hline 45 & Musculium lacustre (O. F. Müller, 1774) & & & $\times$ \\
\hline 46 & Pisidium amnicum (O. F. Müller, 1774) & $x$ & $x$ & $\times$ \\
\hline 47 & Pisidium henslowanum (Sheppard, 1823) & & $x$ & $x$ \\
\hline 48 & Pisidium supinum A. Schmidt, 1851 & & $\times$ & $\times$ \\
\hline 49 & Pisidium milium Held, 1838 & & $\times$ & \\
\hline 50 & Pisidium subtruncatum Malm, 1855 & & $x$ & $\times$ \\
\hline 51 & Pisidium nitidum Jenyns, 1832 & & $\times$ & $\times$ \\
\hline 52 & Pisidium crassum Stelfox, 1918 & & $\times$ & $\times$ \\
\hline 53 & Pisidium obtusale (Lamarck, 1818) & $x$ & & \\
\hline 54 & Pisidium casertanum (Poli, 1791) & $x$ & $\times$ & $\times$ \\
\hline 55 & Pisidium hibernicum Westerlund, 1894 & & $\times$ & \\
\hline 56 & Pisidium pulchellum Jenyns, 1832 & & $\times$ & \\
\hline \multirow[t]{2}{*}{57} & Pisidium moitessierianum Paladilhe, 1866 & & $\times$ & \\
\hline & total & 39 & 43 & 38 \\
\hline
\end{tabular}

have been present in the Krutynia permanently for at least several dozen years.

Six of the species listed in the two earlier papers were not recorded in this study. These included Valvata cristata, Marstoniopsis scholtzi, Bithynia leachii, Physa fontinalis, Gyraulus crista and Hippeutis complanatus. Most of them were described by BERGER (1960) as infrequent (only empty shells of $H$. complanatus were found in the Krutynia). Four other species (Potamopyrgus antipodarum, Stagnicola corvus, Sphaerium rivicola and Musculium lacustre) were recorded only in this study.

Neither HILBERT (1913) nor BERGER (1960) specified in which part of the river they collected their material. Detailed information on the studies in the lower section of the Krutynia was given by BERGER (1962) in his paper on the occurrence of bivalves of the family Sphaeriidae.

The malacofauna of the Krutynia is rich, though some rivers in Poland hold more species, for example the Grabia with its 50 species (PIECHOCKI 1969) or the Pasłęka with 43 species (PIECHOCKI 1972). The high species richness in the former case may result from the fact that the studies involved not only the river but also the stagnant water bodies in its floodplain. We analysed only the proper river, without the lakes it flows through. Differences in species richness between rivers of similar length may result from their water quality.

The content of nitrogen, phosphorus and organic matter in bottom sediments of the Krutynia was relatively small and similar to that in other lowland rivers (e.g. SVEDESEN et al. 1993, CLARKE \& WHARTON 2001, KORYCIŃSKA \& KRÓLAK 2007). Slightly higher content of nitrogen and organic matter in sites 8 and 9 resulted from the character of substratum, which, apart from sand, stones and gravel, contained large amounts of mud accumulating in stagnant river coves.

The correlation between the content of nitrogen, phosphorus, and organic matter in bottom sediments and the number of species was not statistically significant. Chemical parameters do not always affect significantly the qualitative and quantitative composition of the malacofauna. This was confirmed by the studies of STRZELEC (1993) carried out in several dozen anthropogenic water bodies in Silesia. From among many analysed physical and chemical parameters of the water $\left(\mathrm{pH}\right.$, total hardness $\mathrm{Ca}^{2+}, \mathrm{Cl}^{-}, \mathrm{Fe}^{2+}, \mathrm{N}_{-} \mathrm{NH}_{4}$, $\mathrm{N}-\mathrm{NO}_{3}, \mathrm{BOD}_{5}$ ) only the content of $\mathrm{Mg}^{2+}$ and $\mathrm{SO}_{4}{ }^{2-}$ seemed to affect the number of species (STRZELEC 1993).

Individual sites in the Krutynia River differed, apart from chemical properties of bottom sediments, also in the type of substratum, plant cover and the distance from the nearby lake. Only the distance from the lake outlet appeared to be significant for species richness. The sites located close to lakes were characterised by higher richness and diversity than those situated far from lakes. A maximum of 10 species was noted in the sites situated far from lakes. The sites closer to lakes had a much richer malacofauna, with the number of species often exceeding 10. These sites may be regarded as ecotone habitats where both riverine and lacustrine species co-exist (e.g. WOTTON 1988, RICHARDSON \& MACKAY 1991, LEWANDOWSKI 1996b).

To obtain a comprehensive picture of the malacofauna of the Krutynia, also the lakes should be studied. The river flows through 17 lakes and this may undoubtedly modify species composition of molluscs in the river itself. 


\section{ACKNOWLEDGEMENTS}

The authors wish to express their gratitude to EWA JURKIEWICZ-KARNKOWSKA for her help in determin-

\section{REFERENCES}

BAJKIEWICZ-GRABOWSKA E. 1996. Odporność jezior systemu rzeczno-jeziornego Krutyni (Pojezierze Mazurskie) i podatność ich zlewni na degradację. Zeszyty Naukowe Komitetu „Człowiek i Środowisko” 13: 43-59.

BERGER L. 1960. Badania nad mięczakami (Mollusca) Pojezierza Mazurskiego. Bad. Fizjogr. Pol. Zach. 6: 7-49.

BERGER L. 1962. Uwagi o rozmieszczeniu małźów Sphaeriidae w Krutyni na Pojezierzu Mazurskim. Fragm. Faun. 10: 1-9.

BERNATOWICZ S., Wolny P. 1974. Botanika dla limnologów i rybaków. PWRiL, Warszawa.

Clarke S. J., WharTON G. 2001. Sediment nutrient characteristics and aquatic macrophytes in lowland English rivers. Sci. Total Environ. 266: 103-112.

Hilbert R. 1913. Die Molluskenfauna des Kruttinflusses, Kr. Sensburg, Ostpr. Arch. Naturgesch. 12: 91-96.

Hillbricht-IlkowSKA A., WiŚNIEWSKI R. J. (eds.) 1996. Funkcjonowanie systemów rzeczno-jeziornych w krajobrazie pojeziernym: rzeka Krutynia (Pojezierze Mazurskie). Zeszyty Naukowe Komitetu „Człowiek i Środowisko" 13: 1-461.

JAKUBIK B., LEWANDOWSKI K. 2008. Molluscs of the outlet stretch of the Krutynia River (Masurian Lakeland). Annales UMCS 63: 53-58.

KORYCIŃSKA M., KRÓLAK E. 2007. The effect of selected environmental factors on the occurrence of macroinvertebrates in the Osownica River. Oceanol. Hydrobiol. Stud. 36: 55-61.

LEWANDOWSKI K. 1996a. Występowanie Dreissena polymorpha (Pall.) oraz małży z rodziny Unionidae w systemie rzeczno-jeziornym Krutyni (Pojezierze Mazurskie). Zeszyty Naukowe Komitetu „Człowiek i Środowisko” 13: 173-185.

LEWANDOWSKI K. 1996b. Bentos rzeczno-jeziornych stref przejściowych systemu rzeki Krutyni (Pojezierze Mazur- ing bivalves of the genus Pisidium, to ELŻBIETA BIARDZKA for her help in chemical analyses and to LECH KUFEL for translating this text.

skie). Zeszyty Naukowe Komitetu „Człowiek i Środowisko" 13: 303-312.

ŁOMNICKI A. 1995. Wprowadzenie do statystyki dla przyrodników. Wydawnictwo Naukowe PWN, Warszawa.

MARCZENKO Z. 1979. Spektrofotometryczne oznaczanie pierwiastków. PWN, Warszawa.

MARCZEWSKi E., SteinHAus H. 1959. O odległości systematycznej biotopów. Zastosow. Matem. 4: 195-203.

MARGALEF R. 1958. Information theory in ecology. Gen. Syst. 3: 31-76.

PIECHOCKI A. 1969. Mięczaki (Mollusca) rzeki Grabi i jej terenu zalewowego. Fragm. Faun. 15: 111-197.

PIECHOCKI A. 1972. Materiały do poznania mięczaków (Mollusca) rzeki Pasłęki. Fragm. Faun. 18: 121-139.

PIECHOCKI A. 1989. The Sphaeriidae of Poland (Bivalvia, Eulamellibranchiata). Ann. Zool. 42: 249-320.

RiCHARDSON J. S., MACKAY R. J. 1991. Lake outlets and the distribution of filter feeders: an assessment of hypotheses. Oikos 62: 370-380.

STRZELEC M. 1993. Ślimaki (Gastropoda) antropogenicznych środowisk wodnych Wyżyny Śląskiej. Uniwersytet Śląski, Katowice.

Svendsen L. M., RebSdorf A., Norberg P. 1993. Comparison of methods for analysis of organic and inorganic phosphorus in river sediment. Water Res. 27: 77-83.

WOTTON R. S. 1988. Very high secondary production at a lake outlet. Freshw. Biol. 20: 341-346.

Received: November 11th, 2009

Revised: September 12th, 2010

Accepted: December 4th, 2010 